\title{
Some Axi-Symmetric Determinants with Integers for Elements.
}

\author{
By J. J. NAssau, Ph.D.
}

(Received 20th January 1924. Read 7th March 19:4).

1. B. Pascal and others have studied the properties of the table : *

$\begin{array}{rrrrrrr}0 & 1 & 1 & 1 & . & . & . \\ 1 & 2 & 3 & 4 & . & . & . \\ 1 & 3 & 6 & 10 & . & . & . \\ 1 & 4 & 10 & 20 & . & . & .\end{array}$

'The purpose of this paper is to study: (a) determinants formed from the above table together with principal minors bordered by the first row and column of the above table in which any number is substituted for the zero element; $(b)$ the sum of the signed primary minors of these determinants

2. Denote the axi-symmetric determinant

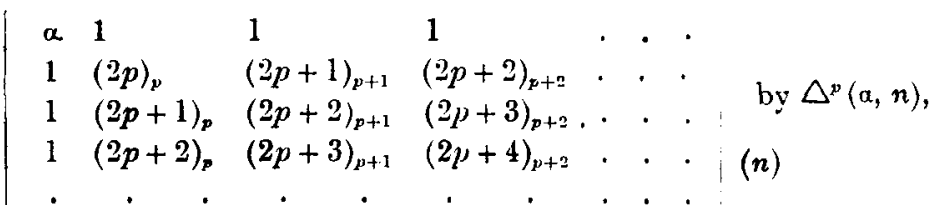

where $(r)_{0}$ stands for the combinatory number

$$
\frac{r(r-1) \ldots \ldots(r-s+1)}{s !} .
$$

Also

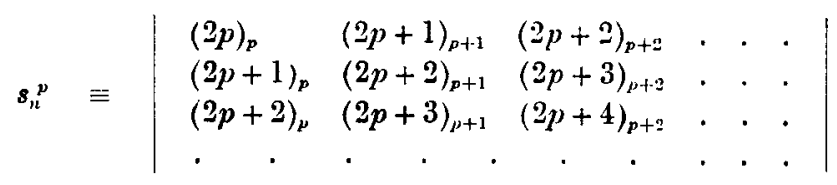

- Dicksur. History of the Theory of Numbers, Vol. II., p. 9. 
The sum of the signed primary minors of any determinant will be denoted by attaching $v$ in front of the determinant.

3. Some fundamental propositions in regard to the sum of the signed primary minors of a determinant are : *

(I) An expression for the negative sum of the signed primary minors of any determinant is got by taking a determinant of the next higher order, whose first element is zero, with the given determinant for supplementary minor, and whose remaining elements are units.

(II) The sum of the signed primary minors of any determinant is expressible as a determinant of the next lower order, any element $(r, s)$ of the latter being the sum of the signed elements of a two-line minor of the former, viz., the $\operatorname{sum}(r, s)-(r, s+1)-(r+1, s)+(r+1, s+1)$.

Let us now consider $\Delta^{\prime}(0, n)$, that is

$$
\begin{array}{|ccccccc}
0 & 1 & 1 & 1 & . & . & . \\
1 & (2)_{1} & (3)_{2} & (4)_{3} & . & . & . \\
1 & (3)_{1} & (4)_{2} & (5)_{3} & . & . & . \\
1 & (4)_{1} & (5)_{2} & (6)_{3} & . & . & . \\
. & . & . & . & . & . & .
\end{array}
$$

which represents according to $I$ the negative sum of the signed primary minors of $s^{\prime}{ }_{n-1}$. Applying II to $s_{n-1}^{\prime}$ we have

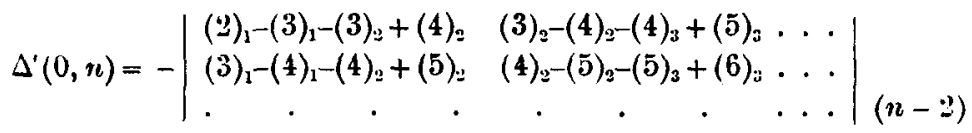

which after making use of the relation $(m)_{\gamma}=(m-1)_{\gamma}+(m-1)_{\gamma-1}$ becomes

$$
\Delta^{\prime}(0, n)=-s^{\prime}
$$

The process being perfectly general we may write

$$
\Delta^{p}(0, n)=-s_{n-2}^{p}
$$

from which we observe that the sum of the signed primary minors of $s_{n}{ }^{\mu}$ is equal to the minor of the last element of its principal diagonal, that is,

$$
\sigma \varepsilon_{n}^{p}=s_{n-1}^{p}
$$

"Moir. The Generating Functiona of Certain Special Doterminanta, Proc. Roy. Soc. Edin., XXIV. 

ations

4. The determinant $s_{n}^{\prime}$ after performing the following oper-

$$
\begin{aligned}
& \operatorname{col}_{n}-\operatorname{col}_{n-1} \quad \operatorname{row}_{n}-\operatorname{row}_{n-1} \\
& \operatorname{col}_{n-1}-\operatorname{col}_{n-2} \text { and } \text { row }_{n-1}-\operatorname{row}_{n-3} \\
& \mathrm{col}_{3}-\mathrm{col}_{1} \quad \operatorname{row}_{2}-\operatorname{row}_{1}
\end{aligned}
$$

takes the form of

that is

$$
\left|\begin{array}{ccccccc}
2 & 1 & 1 & 1 & \cdot & \cdot & \cdot \\
1 & (2)_{1} & (3)_{2} & (4)_{3} & \cdot & \cdot & \cdot \\
1 & (3)_{1} & (4)_{2} & (5)_{3} & \cdot & \cdot & \cdot \\
1 & (4)_{1} & (5)_{2} & (6)_{3} & \cdot & \cdot & \cdot \\
\cdot & \cdot & \cdot & \cdot & \cdot & \cdot & \cdot
\end{array}\right|_{(n)}
$$

and from (1) we obta

$$
s_{n}^{\prime}=\Delta^{\prime}(2, n)
$$

$$
\Delta^{\prime}(0, n+2)=-\Delta^{\prime}(2, n)
$$

5. The evaluation of determinants of the type $\boldsymbol{s}_{n}{ }^{p}$ and therefore their signed primary minors can be effected by considering

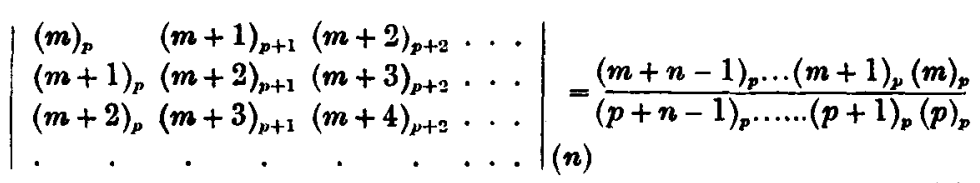

result obtained from Zeipel.* By substituting $m=2 p$ in the above the left side takes the form of the axi-symmetric determinant $a_{n}^{p}$.

When $p=1$ we have

$$
s_{n}^{\prime}=\frac{(n+1) \ldots(2)}{n \ldots l}=n+1
$$

and for $p=2$ we bave

$$
s_{n}^{2}=\frac{(n+3)_{2} \ldots(4)_{2}}{(n+1)_{2} \ldots(2)_{2}}=\frac{(n+3)(n+2)^{3}(n+1)}{12} .
$$

- Losd's Univ. Arsestrift, II, pp. 1.68 or Morz's Hietory of the Theory of Determinanls, Vol. III, p. 148. 
From which, values for $\Delta^{\prime}(0, n)$ and $\Delta^{\prime}(2, n)$ are readily obtained.

We can readily see that

$$
\Delta^{p}(\alpha, n)=\alpha s_{n-1}^{p}+\Delta^{p}(0, n)
$$

or by (1)

$$
\Delta^{n}(a, n)=\alpha s_{n-1}^{p}-s_{n-2}^{p}
$$

When $\alpha=1$ and $p=1$ and by making use of result obtained in (7) equation (9) becomes

$$
\Delta^{\prime}(1, n)=1
$$

This determinant was first considered by Caldarera.*

Equation (4) can now be obtained by substituting $\alpha=2$ and $p=1$ in (9), that is

$$
\Delta^{\prime}(2, n)=2 n-n+1=s_{n}^{\prime} .
$$

6. From paragraph 3, proposition 1 , we have

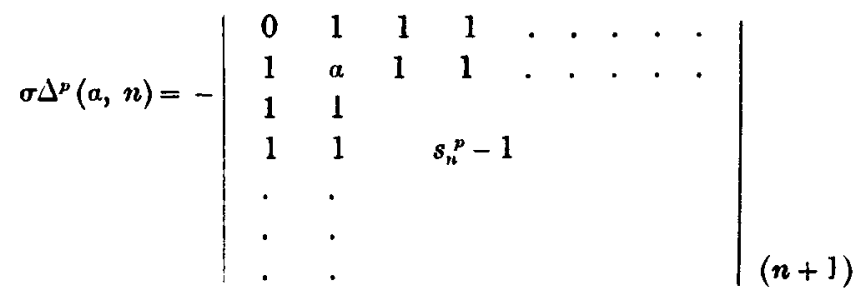

or

Therefore

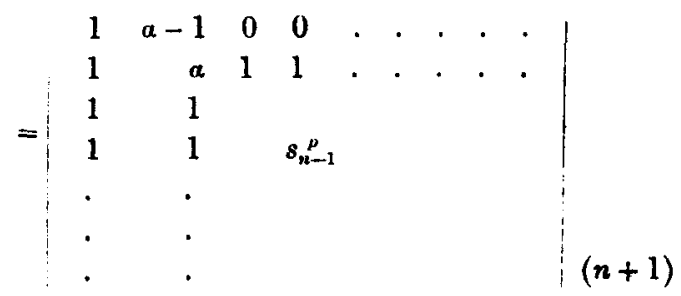

$$
\sigma \Delta^{p}(a, n)=\Delta^{p}(\alpha, n)-(\alpha-1) \Delta^{p}(1, n)
$$

Some special cases:

(a) $\quad a=0$

$$
\sigma \Delta^{p}(0, n)=\Delta^{p}(0, n)+\Delta^{p}(1, n)
$$

- Giornale di Mat., IX., pp., 223-232. 
by (2) and (9)

and by (3)

$$
\begin{aligned}
& =-\boldsymbol{s}_{n-2}^{p}+\boldsymbol{s}_{n-1}^{p}-s_{n-2}^{p} \\
& =s_{n-1}^{p}-2 \delta_{n-2}^{p} \\
& =s_{n-1}^{p}-2 \sigma \delta_{n-1}^{p}
\end{aligned}
$$

Dr Muir * states the above result for the general determinant; however, in the place of the minus sign here given, he has written "together with."

(b) $\quad a=1$

$$
\sigma \Delta^{p}(1, n)=\Delta^{p}(1, n)
$$

which suggests a method of reducing the order of the determinant $\Delta^{p}(1, n)$ by one, by single application of proposition $\Pi$, paragraph 3 , as the determinant so obtained is equal to $\sigma \Delta^{\mu}(1, n)$.

When $p=1$

$$
\sigma \Delta^{\prime}(1, n)=1
$$

(c) $\quad a=2 \quad p=1$

$$
\sigma \Delta^{\prime}(2, n)=\Delta^{\prime}(2, n)=1
$$

as $\Delta^{\prime}(1, n)=1$ from $(10)$.

7. Let us now consider

$$
\Delta^{p}(\alpha, n-1)=\alpha s_{u-2}^{p}-s_{n-3}^{p}
$$

an equation similar to (9) and by dividing both sides by $\alpha$ and adding it to (9) we obtain

$$
\Delta^{p}(\alpha, n)+\frac{1}{a} \Delta^{p}(u, n-1)=\alpha s_{n-1}^{p}-\frac{1}{\alpha} s_{n-3}^{p}
$$

Similarly

$$
\Delta^{p}(a, n-2)=a s_{n \rightarrow 3}^{p}-s_{n+1}^{p} ;
$$

dividing by $a^{2}$ and adding the result to the last equation we get

$$
\Delta^{\nu}(\alpha, n)+\frac{1}{\alpha} \Delta^{\nu}(\alpha, n-1)+\frac{1}{\alpha^{2}} \Delta^{\nu}(\alpha, n-2)=\alpha \delta_{n-1}^{p}-\frac{1}{a^{2}} s_{n-4}^{\mu} .
$$

* Morr. The Generating Functions of Certain Special Determinants, Proc. R.S.L., XXIV, p. 391, proposition XIV. 
Repeating the process $n-2$ times we obtain

$$
\Delta^{p}(\alpha, n)+\frac{1}{\alpha} \Delta^{p}(\alpha, n-1)+\ldots+\frac{1}{\alpha^{n-2}} \Delta^{p}(\alpha, 2)=\alpha s_{n-1}^{p}-\frac{1}{\alpha^{n-2}} s_{n}^{p} .
$$

But $s_{a}^{p}=1, \Delta^{\mu}(\alpha, 1)=\alpha$ and by transferring the last term to the left side of equation it takes the form of

$$
\Delta^{p}(a, n)+\frac{1}{a} \Delta^{p}(\alpha, n-1)+\ldots+\frac{1}{a^{n-1}} \Delta^{p}(\alpha, 1)=\alpha s_{n-1}^{p}
$$

Substituting $a=1$ in equation (12) we get the determinant $\Delta^{n}(1, n)$ plus the minor of last element of the principal diagonal of $\Delta^{p}(1, n)$ plus the minor of the last two elements of the principal diagonal of the same determinant, and so on, up to the minor of the last $n-1$ elements of $\Delta^{p}(1, n)$ equal to the minor of the first diagonal element of the same determinant. 